\title{
Dyakonov-Tamm waves guided jointly by an ordinary, isotropic, homogeneous, dielectric material and a hyperbolic, dielectric, structurally chiral material
}

\author{
Akhlesh Lakhtakia and Muhammad Faryad \\ Pennsylvania State University, Department of Engineering Science and Mechanics, \\ Nanoengineered Metamaterials Group (NanoMM), University Park, PA 16802-6812, USA
}

\begin{abstract}
The planar interface of an ordinary, isotropic, homogeneous, dielectric material and a hyperbolic, dielectric, structurally chiral material can support the propagation of one or multiple Dyakonov-Tamm waves, at a specified frequency and along a specified direction in the interface plane. When multiple Dyakonov-Tamm waves can exist, they differ in phase speed, propagation length, degree of localization to the interface, and spatial profiles of the associated electromagnetic fields. Dependence on the relative permittivity scalar of the isotropic partnering material suggests exploitation for optical sensing of analytes.
\end{abstract}

\section{Introduction}

The Dyakonov wave is an electromagnetic surface wave guided by the planar interface of two homogenous dielectric materials, at least one of which is anisotropic. The existence of the Dyakonov wave was predicted in the 1980s [1, 2] and experimentally verified about five years ago [3]. The Tamm wave is guided by the planar interface of two isotropic dielectric materials, at least one of which is periodically nonhomogeneous in the direction normal to the interface. Predicted in 1977 [4], Tamm waves were experimentally observed shortly thereafter [5] and are being applied for optical sensing of analytes [6, 7].

Whereas the anisotropy of a partnering material provides the Dyakonov wave a sensitivity to the direction of propagation [8], the periodic nonhomogeneity of a partnering material is responsible for multiple Tamm waves to propagate in any direction [9]. Both of these attributes are combined in Dyakonov-Tamm waves, whose propagation is guided by the interface of two dielectric materials, one of which must be anisotropic and periodically nonhomogeneous normal to the interface $[10,11]$. Recently, the existence of these Dyakonov-Tamm waves was confirmed experimentally using a prism-coupled configuration $[12]$. 
In the foregoing papers on Dyakonov-Tamm waves, one partnering material is an ordinary, isotropic, homogeneous, dielectric material with a purely real relative permittivity $\varepsilon_{i s o}>0$, whereas the real part of the relative permittivity dyadic $\underline{\underline{\varepsilon}}_{a n i}$ of the anisotropic, periodically nonhomogeneous, dielectric partnernering material is positive definite, i.e., all three eigenvalues of $\underline{\underline{\varepsilon}}_{a n i}$ have positive real parts [13]. What would happen if $\operatorname{Re}\left(\underline{\underline{\varepsilon}}_{\text {ani }}\right)$ were indefinite, i.e., if either one or two of its eigenvalues had negative real parts, the remaining having positive real parts? Such materials are nowadays called hyperbolic materials. Homogeneous hyperbolic materials exist in nature $[14,15,16]$ and have also been manufactured $[17,18]$. Periodically nonhomogeneous hyperbolic materials appear very likely to be manufacturable [19] using physical-vapor-deposition techniques [20, 21].

In this Letter, we present the results of our investigations on Dyakonov-Tamm waves guided by the planar interface of an ordinary, isotropic, homogenous, dielectric material and a hyperbolic, dielectric, structurally chiral material which is nonhomogeneous in the direction normal to the interface. Section 2 briefly describes the relevant one-point boundaryvalue problem. Numerical results are provided and discussed in Sec. 3. An $\exp (-i \omega t)$ dependence on time $t$ is implicit, with $i=\sqrt{-1}$ and $\omega$ denoting the angular frequency.

\section{Theoretical Preliminaries}

The one-point boundary-value problem of Dyakonov-Tamm-wave propagation is as follows: Suppose that the plane $z=0$ is the interface between the two chosen partnering materials. The half space $z<0$ is occupied by an ordinary, isotropic, homogenous, dielectric material with relative permittivity $\varepsilon_{i s o}$ such that $\operatorname{Re}\left(\varepsilon_{i s o}\right)>0$ and $\operatorname{Im}\left(\varepsilon_{i s o}\right)=0$. The half space $z>0$ is occupied by a hyperbolic, dielectric, structurally chiral material with relative permittivity dyadic [19]

$$
\underline{\underline{\varepsilon}}_{a n i}(z)=\varepsilon_{0} \underline{\underline{S}}_{z}(z) \cdot \underline{\underline{S}}_{y}(\chi) \cdot\left(\varepsilon_{a} \hat{\mathbf{u}}_{z} \hat{\mathbf{u}}_{z}+\varepsilon_{b} \hat{\mathbf{u}}_{x} \hat{\mathbf{u}}_{x}+\varepsilon_{c} \hat{\mathbf{u}}_{y} \hat{\mathbf{u}}_{y}\right) \cdot \underline{\underline{S}}_{y}^{-1}(\chi) \cdot \underline{\underline{S}}_{z}^{-1}(z)
$$

where the direction of nonhomogeneity is parallel to the $z$ axis; the Cartesian unit vectors

are identified as $\hat{\mathbf{u}}_{x}, \hat{\mathbf{u}}_{y}$, and $\hat{\mathbf{u}}_{z}$; and $\varepsilon_{0}$ is the permittivity of free space; the periodic nonhomogeneity is expressed through the rotation dyadic

$$
\underline{S}_{z}(z)=\hat{\mathbf{u}}_{z} \hat{\mathbf{u}}_{z}+\left(\hat{\mathbf{u}}_{x} \hat{\mathbf{u}}_{x}+\hat{\mathbf{u}}_{y} \hat{\mathbf{u}}_{y}\right) \cos \left(\frac{h \pi z}{\Omega}\right)+\left(\hat{\mathbf{u}}_{y} \hat{\mathbf{u}}_{x}-\hat{\mathbf{u}}_{x} \hat{\mathbf{u}}_{y}\right) \sin \left(\frac{h \pi z}{\Omega}\right),
$$

with $2 \Omega$ as the period and either $h=+1$ for structural right-handedness or $h=-1$ for structural left-handedness; the dyadic

$$
\underline{S}_{y}(\chi)=\left(\hat{\mathbf{u}}_{x} \hat{\mathbf{u}}_{x}+\hat{\mathbf{u}}_{z} \hat{\mathbf{u}}_{z}\right) \cos \chi+\left(\hat{\mathbf{u}}_{z} \hat{\mathbf{u}}_{x}-\hat{\mathbf{u}}_{x} \hat{\mathbf{u}}_{z}\right) \sin \chi+\hat{\mathbf{u}}_{y} \hat{\mathbf{u}}_{y}
$$


contains the tilt angle $\chi \in[0, \pi / 2]$ with respect to the $x y$ plane; $\varepsilon_{a}, \varepsilon_{b}$, and $\varepsilon_{c}$ are the three $z$-independent eigenvalues of $\underline{\underline{\varepsilon}}_{a n i}(z)$; and either one or two of these three eigenvalues have negative real parts but the remainder do not.

The electromagnetic field phasors everywhere can be written as [10]

$$
\left.\begin{array}{l}
\mathbf{E}(\mathbf{r})=\mathbf{e}(z) \exp [i q(x \cos \psi+y \sin \psi)] \\
\mathbf{H}(\mathbf{r})=\mathbf{h}(z) \exp [i q(x \cos \psi+y \sin \psi)]
\end{array}\right\}
$$

with the unknown surface wavenumber $q$ and unknown functions $\mathbf{e}(z)$ and $\mathbf{h}(z)$. The angle $\psi \in[0,2 \pi)$ denotes the direction of propagation in the $x y$ plane. As the procedure to obtain and solve a dispersion equation for $q$, and then determine $\mathbf{e}(z)$ and $\mathbf{h}(z)$, for a specific $\psi$ has been described elsewhere in detail [10], it is not repeated here.

\section{Numerical results and discussion}

For illustrative results, we set $\varepsilon_{a}=2.26(1+i \delta), \varepsilon_{b}=3.46(-1+i \delta), \varepsilon_{c}=2.78(1+i \delta)$, $\delta=0.001, h=+1, \Omega=135 \mathrm{~nm}, \chi=\pi / 6$, and $\psi=0$. Without loss of generality, we took the isotropic partner to be free space (i.e., $\left.\varepsilon_{i s o}=1\right)[22]$. Our search was mostly restricted to the regime $\operatorname{Re}(q) / k_{0} \in(1,4]$, where $k_{0}$ is the free-space wavenumber.

At every free-space wavelength $\lambda_{0} \in[600,700] \mathrm{nm}$, we found three solutions $q$ of the dispersion equation. The solutions were organized in three branches, as shown in Fig. 1. Thus, three distinct Dyakonov-Tamm waves differing in phase speed $v_{p h}=\omega / \operatorname{Re}(q)$ and propagation length $\Delta_{\text {prop }}=1 / \operatorname{Im}(q)$ can propagate along the $x$ axis in the interface plane for $\lambda_{0} \in[600,700] \mathrm{nm}$. Dyakonov-Tamm waves on the highest- $v_{p h}$ branch have $v_{p h} \simeq 0.935 c_{0}$, where $c_{0}=\omega / k_{0}$ is the speed of light in free space, and $\Delta_{\text {prop }}$ ranging from $\sim 160 \mathrm{~nm}$ to $\sim 200 \mathrm{~nm}$. Dyakonov-Tamm waves on the lowest- $v_{p h}$ branch have $v_{p h}$ ranging from $\sim 0.23 c_{0}$ to $\sim 0.264 c_{0}$, and $\Delta_{\text {prop }} \simeq 7.0 \mu \mathrm{m}$. Clearly from the presented data, a lower phase speed is associated with higher attenuation along the direction of propagation.

The Dyakonov-Tamm waves on the three branches in Fig. 1 are not only dissimilar in phase speed and propagation length, but the spatial profiles of their field along the $z$ axis also differ. Let us focus on the three solutions of the dispersion equation for $\lambda_{0}=635 \mathrm{~nm}$. Figure 2 presents plots of the magnitudes of the Cartesian components of $\mathbf{e}(z)$ and $\mathbf{h}(z)$ as functions of $z$ for the Dyakonov-Tamm wave with $q=(1.0686+i 0.0006) k_{0}$, Fig. 3 for the Dyakonov-Tamm wave with $q=(1.7507+i 0.0031) k_{0}$, and Fig. 4 for the Dyakonov-Tamm wave with $q=(3.9809+i 0.0139) k_{0}$. On the isotropic side, the degree of localization of the Dyakonov-Tamm wave to the interface is greater if $\operatorname{Re}(q)$ is higher. However, on the anisotropic side of the interface, a clear dependence of localization to the interface does 
not emerge from the three figures. Indeed, of the three Dyakonov-Tamm waves, the one in Fig. 4 is the most strongly localized to the interface, whereas the one in Fig. 3 is the most weakly localized to the interface.

The Dyakonov-Tamm-wave-propagation phenomenon depends strongly on $\psi$. This is exemplied by the plots in Fig. 5 of $v_{p h}$ and $\Delta_{\text {prop }}$ as functions of $\psi \in\left[-90^{\circ}, 90^{\circ}\right]$ when $\lambda_{0}=635 \mathrm{~nm}$ and $\chi=30^{\circ}$. The solutions can be organized in six branches. One branch, with the highest values of $v_{p h}$ and $\Delta_{\text {prop }}$ spans the entire angular regime $\psi \in\left[-90^{\circ}, 90^{\circ}\right]$ available for propagation. Each of the remaining five branches spans a finite range of $\psi$. Although the five branches appear to be clustered together, the phase speeds range from $0.14 c_{0}$ to $0.58 c_{0}$ and the propagation lengths from $\sim 2 \mu \mathrm{m}$ to $\sim 34 \mu \mathrm{m}$, which are quite wide ranges. Either one or two or three Dyakonov-Tamm waves can propagate in the interface plane at an angle $\psi$ with respect to the $x$ axis. Let us also note that, because of chiral symmetry about the $z$ axis, the solutions of the dispersion equation are the same for $\psi$ and $\psi \pm 180^{\circ}$.

The Dyakonov-Tamm-wave-propagation phenomenon also depends strongly on $\varepsilon_{i s o}$. Suppose that free space, the isotropic partnering material for Figs. 2-4, were to be replaced by a dielectric material with $\varepsilon_{i s o}=2.25$. Then the dispersion equation has just only two solutions $-q=(1.9227+i 0.0040) k_{0}$ and $q=(4.2766+i 0.0150) k_{0}$-instead of the three solutions for $\varepsilon_{i s o}=1$. The large changes between the solutions for the two values of $\varepsilon_{\text {iso }}$ chosen here strongly suggest the potential of Dyakonov-Tamm waves supported by a partnering material that is hyperbolic, dielectric, and structurally chiral for optical sensing of analytes in a prism-coupled configuration, just as both surface-plasmon-polariton waves [23] and Tamm waves $[6,7]$ are being used by technoscientists.

Acknowledgments. A.L. is grateful to the Charles Godfrey Binder Endowment at the Pennsylvania State University for the financial support of his ongoing research. M.F. was supported by Grant No. DMR-1125591 from the U.S. National Science Foundation.

\section{References}

[1] Marchevskiï, F.N.; Strizhevskii, V.L.; Strizhevskii, S.V. Sov. Phys. Solid State 1984, 26, 911-912.

[2] D’yakonov, M.I. Sov. Phys. JETP 1988, 67, 714-716.

[3] Takayama, O.; Crasovan, L.; Artigas, D.; Torner, L. Phys. Rev. Lett. 2009, 102, 043903, 
[4] Yeh, P.; Yariv, A.; Hong, C.S. J. Opt. Soc. Am. 1977, 67, 423-438.

[5] Yeh, P.; Yariv, A.; Cho, A.Y. Appl. Phys. Lett. 1978, 32, 104-105.

[6] Shinn, M.; Robertson, W.M. Sens. Actuat. B: Chem. 2006, 105, 360-364.

[7] Konopsky, V.N.; Alieva, E.V. Anal. Chem. 2007, 79, 4729-4735.

[8] Takayama, O.; Crasovan, L.C.; Johansen, S.K.; Mihalache, D.; Artigas, D.; Torner, L. Electromagnetics 2008, 28, 126-145.

[9] Maab, H.; Faryad, M.; Lakhtakia, A.; J. Opt. Soc. Am. B 2011, 28, 1204-1212.

[10] Lakhtakia, A.; Polo Jr., J.A. it J. Eur. Opt. Soc. Rapid Publ. 2007, 2, 07021.

[11] Faryad, M.; Lakhtakia, A. Phys.Rev.A 2011, 83, 013814.

[12] Pulsifer, D.P.; Faryad, M.; Lakhtakia, A. Phys. Rev. Lett. 2013, 111, 243902.

[13] Lütkepohl, H. Handbook of Matrices; Wiley: Chicester, 1996.

[14] Fisher, R.K.; Gold, R.W. Phys. Rev. Lett. 1969, 22, 1093-1095.

[15] Gerbaux, X.; Tazawa, M.; Hadni, A. Ferroelectrics 1998, 215, 47-63.

[16] Sun, J.; Zhou, J.; Li, B.; Kang, F. Appl. Phys. Lett. 2011, 98, 101901.

[17] Kanungo, J.; Schilling, J. Appl. Phys. Lett. 2010, 97, 021903.

[18] Othman, M. A. K.; Guclu, C.; Capolino, F. J. Nanophotonics 2013, 7, 073089.

[19] Lakhtakia, A. J. Nanophotonics 2014, 8, 083998.

[20] Hawkeye, M.M.; Brett, M.W. J. Vac. Sci. Technol. A 2007, 25, 1317-1335.

[21] Martín-Palma, R.J.; Lakhtakia, A. Nanotechnology-A Crash Course; SPIE: Bellingham, 2010.

[22] Faryad, M.; Lakhtakia, A.; Pulsifer, D.P. J. Opt. Soc. Am. B 2013, 30, 3035-3040.

[23] Homola, J.; Koudela, I.; Yee, S.; Sens. Actuat. B: Chem. 1999, 54, 16-24. 

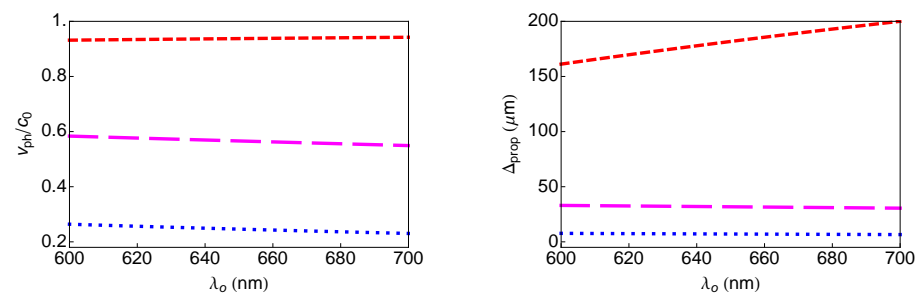

Figure 1: Phase speed $v_{p h}$ and propagation length $\Delta_{\text {prop }}$ as functions of $\lambda_{0}$, when $\varepsilon_{i s o}=1$, $\varepsilon_{a}=2.26(1+i \delta), \varepsilon_{b}=3.46(-1+i \delta), \varepsilon_{c}=2.78(1+i \delta), \delta=0.001, h=+1, \Omega=135 \mathrm{~nm}$, $\chi=\pi / 6$, and $\psi=0$. The solutions of the dispersion equation are organized in three branches.
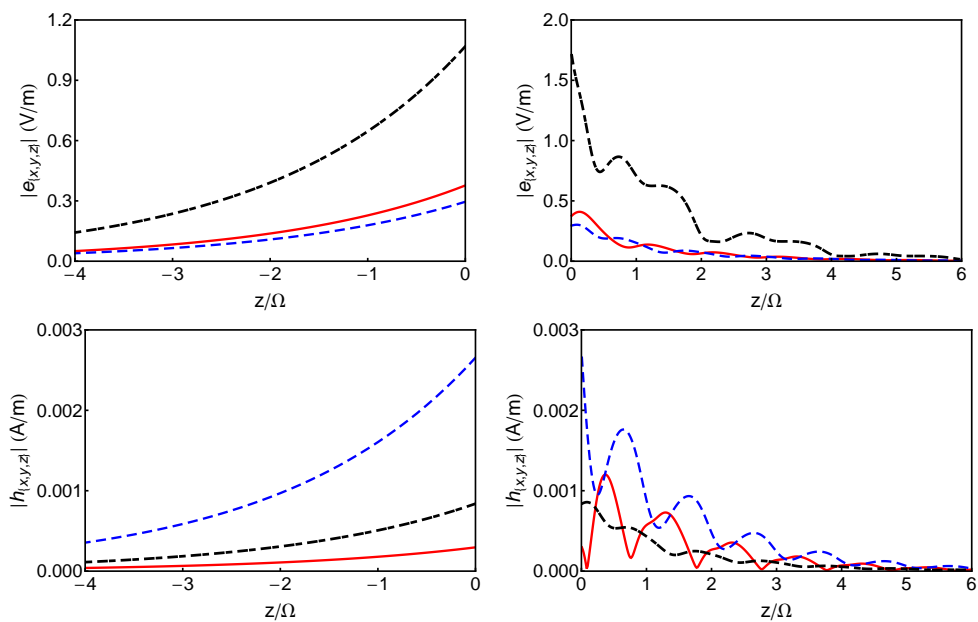

Figure 2: (Color online) Normalized variations with $z$ of the magnitudes of the Cartesian components of $\mathbf{e}(z)$ and $\mathbf{h}(z)$ of an SPP wave when $\lambda_{0}=635 \mathrm{~nm}, \varepsilon_{i s o}=1, \varepsilon_{a}=2.26(1+i \delta)$, $\varepsilon_{b}=3.46(-1+i \delta), \varepsilon_{c}=2.78(1+i \delta), \delta=0.001, h=+1, \Omega=135 \mathrm{~nm}, \chi=\pi / 6$, and $\psi=0$. The $x, y$, and $z$-components are represented by red solid, blue dashed, and black chain-dashed lines, respectively. For this Dyakonov-Tamm wave, $q=(1.0686+i 0.0006) k_{0}$. 

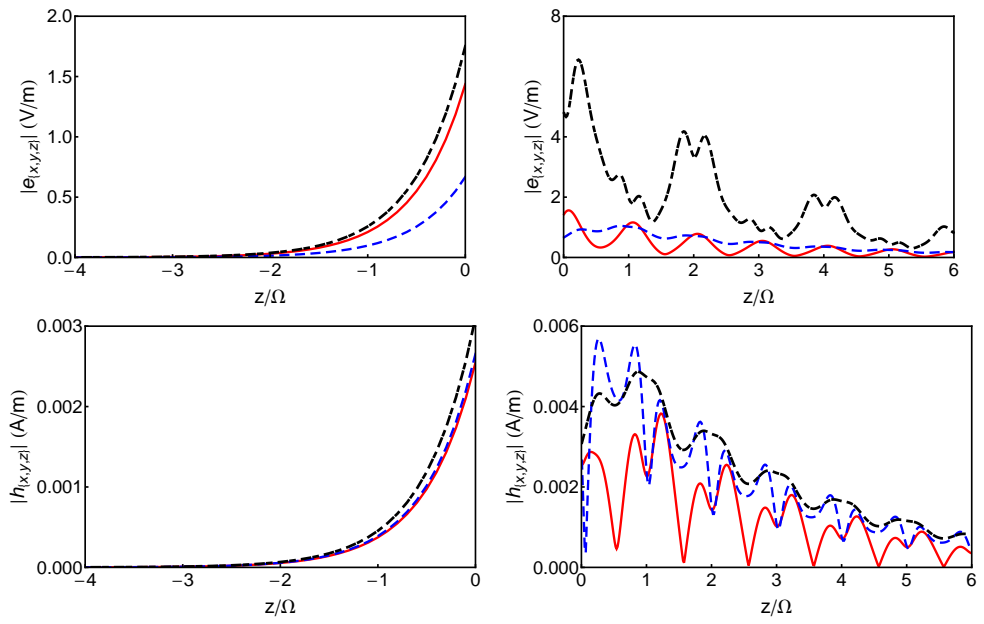

Figure 3: (Color online) Same as Fig. 2, except for the Dyakonov-Tamm wave with $q=$ $(1.7507+i 0.0031) k_{0}$.
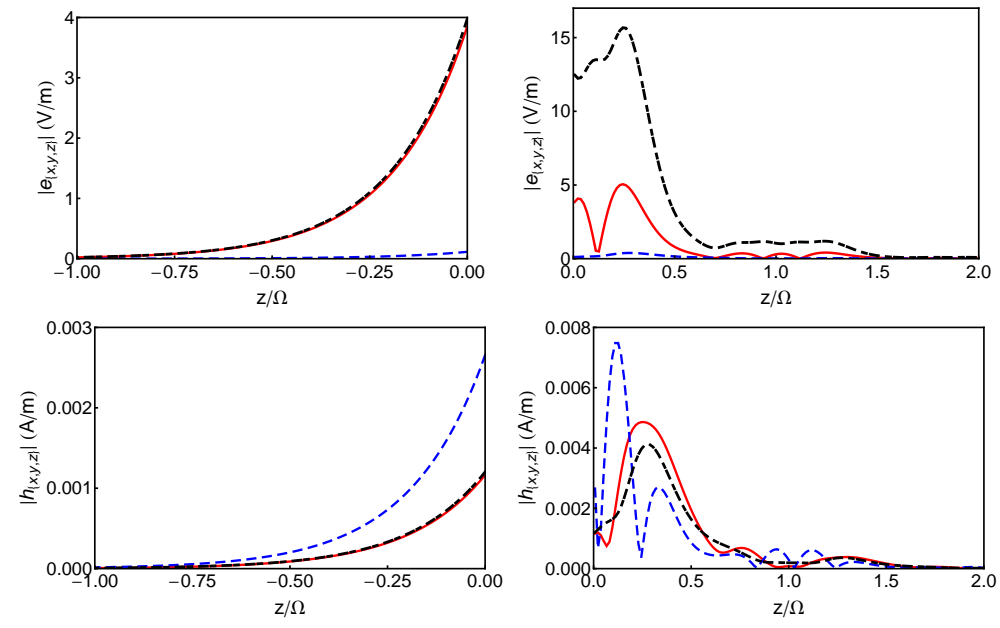

Figure 4: (Color online) Same as Fig. 2, except for the Dyakonov-Tamm wave with $q=$ $(3.9809+i 0.0139) k_{0}$. 

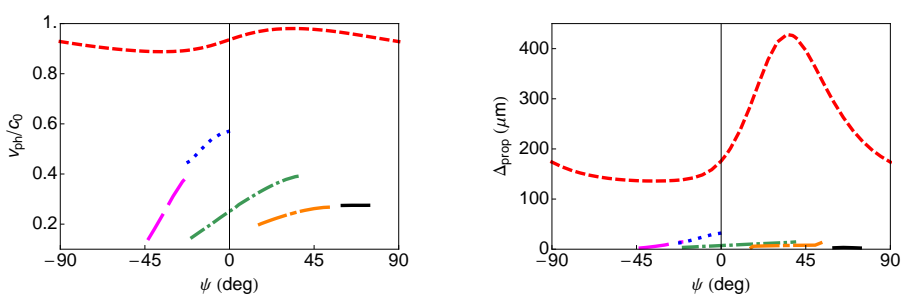

Figure 5: (Color online) Phase speed $v_{p h}$ and propagation length $\Delta_{\text {prop }}$ as functions of $\psi$, when $\lambda_{0}=635 \mathrm{~nm}, \varepsilon_{i s o}=1, \varepsilon_{a}=2.26(1+i \delta), \varepsilon_{b}=3.46(-1+i \delta), \varepsilon_{c}=2.78(1+i \delta)$, $\delta=0.001, h=+1, \Omega=135 \mathrm{~nm}$, and $\chi=30^{\circ}$. The solutions of the dispersion equation are organized in six branches. 